\title{
Existence of solutions for a multivalued boundary value problem with non-convex and unbounded right-hand side
}

\author{
by Diego Averna (Palermo) and \\ Gabriele Bonanno (Reggio Calabria)
}

\begin{abstract}
Let $F:[a, b] \times \mathbb{R}^{n} \times \mathbb{R}^{n} \rightarrow 2^{\mathbb{R}^{n}}$ be a multifunction with possibly non-convex and unbounded values. The main result of this paper (Theorem 1) asserts that, given the multivalued boundary value problem

$$
\left\{\begin{array}{l}
u^{\prime \prime} \in F\left(t, u, u^{\prime}\right), \\
u(a)=u(b)=\vartheta_{\mathbb{R}^{n}},
\end{array}\right.
$$

if an appropriate restriction of the multifunction $F$ has non-empty and closed values and satisfies the lower Scorza Dragoni property and a weak integrable boundedness type condition, then we can substitute the problem $\left(\mathrm{P}_{F}\right)$ with another one $\left(\mathrm{P}_{G}\right)$, with a suitable convex right-hand side $G$, such that every generalized solution of $\left(\mathrm{P}_{G}\right)$ is also a generalized solution of $\left(\mathrm{P}_{F}\right)$ (see also Remark 1 and Corollary 1).

As a consequence of our results, in conjunction with those in [13] and [18], some existence theorems for multivalued boundary value problems are then presented (see Theorem 2, Corollary 2 and Theorem 3).

Finally, some applications are given to the existence of generalized solutions for two implicit boundary value problems (Theorems 4-6).
\end{abstract}

1. Introduction. Let $([a, b], \mathcal{L}, \mu)$ be the Lebesgue measure space on the compact real interval $[a, b] ; \mathbb{R}^{n}$ the euclidean $n$-space, whose zero element is denoted by $\vartheta_{\mathbb{R}^{n}} ; s \in[1, \infty] ; W^{2, s}\left([a, b], \mathbb{R}^{n}\right):=\left\{u:[a, b] \rightarrow \mathbb{R}^{n} \mid u \in\right.$ $\left.C^{1}\left([a, b], \mathbb{R}^{n}\right), u^{\prime} \in A C\left([a, b], \mathbb{R}^{n}\right), u^{\prime \prime} \in L^{s}\left([a, b], \mathbb{R}^{n}\right)\right\} ; F:[a, b] \times \mathbb{R}^{n} \times \mathbb{R}^{n} \rightarrow$ $2^{\mathbb{R}^{n}}$ a multifunction.

Consider the problem

$\left(\mathrm{P}_{F}\right)$

$$
\left\{\begin{array}{l}
u^{\prime \prime} \in F\left(t, u, u^{\prime}\right) \\
u(a)=u(b)=\vartheta_{\mathbb{R}^{n}}
\end{array}\right.
$$

1991 Mathematics Subject Classification: 34A60, 34B15, 34A09.

Key words and phrases: multivalued differential inclusions, boundary value problems, non-convex and unbounded right-hand side, directional continuous selections, implicit equations.

This research was supported by $60 \%$ MURST. 
A function $u:[a, b] \rightarrow \mathbb{R}^{n}$ is said to be a generalized solution of the problem $\left(\mathrm{P}_{F}\right)$ in $W^{2, s}\left([a, b], \mathbb{R}^{n}\right)$ if $u \in W^{2, s}\left([a, b], \mathbb{R}^{n}\right), u(a)=u(b)=\vartheta_{\mathbb{R}^{n}}$, and $u^{\prime \prime}(t) \in F\left(t, u(t), u^{\prime}(t)\right)$ a.e. in $[a, b]$.

This paper is arranged as follows. After some notations and preliminary results given in Section 2, in Section 3 we prove our main result (Theorem 1) which states that, if $F(t, x, z)$ is a multifunction, with possibly non-convex and unbounded values, such that an appropriate restriction of $F$ satisfies the lower Scorza Dragoni property and a weak integrable boundedness type condition with a function $m \in L^{s}\left([a, b], \mathbb{R}_{0}^{+}\right)$, then there exists another multifunction $G:[a, b] \times \mathbb{R}^{n} \times \mathbb{R}^{n} \rightarrow 2^{\mathbb{R}^{n}}$, with non-empty, closed and convex values, such that $G(\cdot, x, z)$ is measurable, $G(t, \cdot, \cdot)$ has closed graph, $G$ is integrably bounded by $m$, and every generalized solution of the problem

$$
\left\{\begin{array}{l}
u^{\prime \prime} \in G\left(t, u, u^{\prime}\right) \\
u(a)=u(b)=\vartheta_{\mathbb{R}^{n}},
\end{array}\right.
$$

in $W^{2, s}\left([a, b], \mathbb{R}^{n}\right)$ is also a generalized solution of $\left(\mathrm{P}_{F}\right)$ in $W^{2, s}\left([a, b], \mathbb{R}^{n}\right)$ (see also Remark 1 and Corollary 1).

The technical approach consists in the substitution of the multifunction $F$ with another one $H$, which is integrably bounded by $m$ and has the lower Scorza Dragoni property, and in the use of Bressan's directional continuous selections ([6]) in order to obtain $G$ by means of a convexification.

In Section 4, some existence theorems for problem $\left(\mathrm{P}_{F}\right)$ follow as a simple consequence of our theorems and Theorem 2.1 of [13] (see Theorem 2 and Corollary 2). They both improve Theorem 3 of [8]. Moreover, by using a result of [18] and our Theorem 2, an existence theorem for the problem

$$
\left(\mathrm{P}_{F \circ G}\right) \quad\left\{\begin{array}{l}
u^{\prime \prime} \in F\left(G\left(t, u, u^{\prime}\right)\right), \\
u(a)=u(b)=\vartheta_{\mathbb{R}^{n}},
\end{array}\right.
$$

is given (Theorem 3), where the multifunction $F \circ G$ is not required to be lower or upper semicontinuous, and its values can be non-convex, non-closed and unbounded (see also Remark 4).

In Section 5, some applications are given of our results to the existence of generalized solutions in $W^{2, s}\left([a, b], \mathbb{R}^{n}\right)$ for a boundary value problem for second-order implicit equations $f\left(t, u, u^{\prime}, u^{\prime \prime}\right)=0$. Usually, in the literature, very strong conditions are required for $f\left(t, u, u^{\prime}, \cdot\right)$ to assure existence of solutions for such a problem (such as lipschitzianity, with Lipschitz constant strictly less than 1). The first attempt to obtain existence theorems where rather general conditions on the function $f$ with respect to the last variable are required seems to be [14], to which we refer for other bibliographical references.

We give three theorems. 
The first one (Theorem 4) is an existence theorem for the boundary value problem

$$
\left\{\begin{array}{l}
f\left(t, u, u^{\prime}, u^{\prime \prime}\right)=0 \\
u(a)=u(b)=\vartheta_{\mathbb{R}^{n}}
\end{array}\right.
$$

where, given a non-empty, connected, locally connected, but possibly nonclosed and unbounded subset $Y$ of $\mathbb{R}^{n}, f:[a, b] \times \mathbb{R}^{n} \times \mathbb{R}^{n} \times Y \rightarrow \mathbb{R}$ is a function which, besides other conditions, is continuous in its last variable (for suitable values of $\left.\left(t, u, u^{\prime}\right)\right)$ and satisfies with respect to the other variables a condition weaker than the Scorza Dragoni property.

The second one (Theorem 5) is another existence theorem for the boundary value problem $\left(\mathrm{P}_{f}^{i}\right)$, where $Y$ is a non-empty, bounded, connected and locally connected, but possibly non-closed subset of $\mathbb{R}^{n}$, and $f$ is again continuous in $u^{\prime \prime}$. This theorem, just as Theorem 2.1 of [14], in which $Y$ is also closed, gives existence of solutions in $W^{2, \infty}\left([a, b], \mathbb{R}^{n}\right)$.

The last one (Theorem 6 ) is an existence theorem for the boundary value problem

$$
\left(\mathrm{P}_{f, g}^{i}\right) \quad\left\{\begin{array}{l}
f\left(u^{\prime \prime}\right)=g\left(t, u, u^{\prime}\right), \\
u(a)=u(b)=\vartheta_{\mathbb{R}^{n}},
\end{array}\right.
$$

where, given a non-empty subset $Y$ of $\mathbb{R}^{n}, f: Y \rightarrow \mathbb{R}$ is not required to be continuous, and a suitable restriction of $g:[a, b] \times \mathbb{R}^{n} \times \mathbb{R}^{n} \rightarrow \mathbb{R}$ has the Scorza Dragoni property. Theorem 6 improves Theorem 2.2 of [14], in which the continuity of $f$ and $g$ is required, $Y$ is a non-empty, compact, connected and locally connected subset of $\mathbb{R}^{n}$, and only generalized solutions in $W^{2, \infty}\left([a, b], \mathbb{R}^{n}\right)$ can be obtained.

Finally, we give an example which shows that our Theorems 4 and 6 can be used to obtain existence of solutions also for boundary value problems with no solutions in $W^{2, \infty}\left([a, b], \mathbb{R}^{n}\right)$.

2. Notations and preliminaries. Let $A, B$ be two non-empty sets. A multifunction $\Phi: A \rightarrow 2^{B}$ is a function from $A$ into the family of all subsets of $B$. The graph of $\Phi$ is the set $\operatorname{gr}(\Phi):=\{(a, b) \in A \times B: b \in \Phi(a)\}$. If $\Omega$ is a subset of $B$, we put $\Phi^{-}(\Omega):=\{a \in A: \Phi(a) \cap \Omega \neq \emptyset\}$ and $\Phi^{+}(\Omega):=\{a \in A: \Phi(a) \subset \Omega\}$. If $C$ is a non-empty subset of $A$, we put $\Phi(C):=\bigcup_{c \in C} \Phi(c)$, and we denote by $\Phi_{\mid C}$ the restriction of $\Phi$ to $C$.

If $\left(A, \tau_{A}\right)$ is a topological space and $E \subset A$, then $\operatorname{int}(E)$ and $\bar{E}$ denote, as usual, the interior and the closure of the set $E$ respectively; $\mathcal{B}(A)$ denotes the $\sigma$-algebra generated by $\tau_{A}$.

If $\left(B, \tau_{B}\right)$ is a topological space, then $\bar{\Phi}$ denotes the multifunction from $A$ into $2^{B}$ defined by $\bar{\Phi}(a)=\overline{\Phi(a)}$.

If $\left(A, \mathcal{F}_{A}\right)$ is a measurable space and $\left(B, \tau_{B}\right)$ a topological space, we say

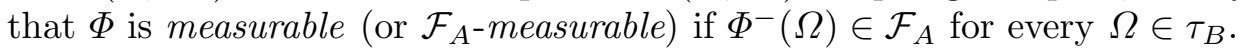


If $\left(A, \tau_{A}\right)$ and $\left(B, \tau_{B}\right)$ are two topological spaces, we say that $\Phi$ is lower (resp. upper) semicontinuous if $\Phi^{-}(\Omega) \in \tau_{A}$ (resp. $\Phi^{+}(\Omega) \in \tau_{A}$ ) for every $\Omega \in \tau_{B} ; \Phi$ is said to be continuous if it is simultaneously lower and upper semicontinuous. We say that a multifunction $\Psi:[a, b] \times A \rightarrow 2^{B}$ has the lower Scorza Dragoni property if for every $\varepsilon>0$ there exists a compact set $T_{\varepsilon} \subset[a, b]$, with $\mu\left([a, b] \backslash T_{\varepsilon}\right)<\varepsilon$, such that $\Psi_{\mid T_{\varepsilon} \times A}$ is lower semicontinuous; we say that a function $f:[a, b] \times A \rightarrow B$ has the Scorza Dragoni property if for every $\varepsilon>0$ there exists a compact set $T_{\varepsilon} \subset[a, b]$, with $\mu\left([a, b] \backslash T_{\varepsilon}\right)<\varepsilon$, such that $f_{\mid T_{\varepsilon} \times A}$ is continuous.

Let $(A, \varrho)$ be a metric space. For every $a \in A$ and every $r \geq 0$, we denote by $B_{\varrho}(a, r):=\left\{a^{\prime} \in A: \varrho\left(a, a^{\prime}\right) \leq r\right\}$ the closed ball of center $a$ and radius $r$ and by $B_{\varrho}^{\circ}(a, r):=\left\{a^{\prime} \in A: \varrho\left(a, a^{\prime}\right)<r\right\}$ the corresponding open ball. If $x \in A$ and $C$ is a non-empty subset of $A$, we put $\varrho(x, A):=\inf \{\varrho(x, c)$ : $c \in C\}$. As usual, when the metric is clear from the context, we use the notations $B(a, r)$ and $B^{\circ}(a, r)$ respectively.

For all $(t, \sigma) \in[a, b] \times[a, b]$, put

$$
K(t, \sigma):= \begin{cases}\frac{(b-t)(\sigma-a)}{b-a} & \text { if } a \leq \sigma \leq t \leq b, \\ \frac{(b-\sigma)(t-a)}{b-a} & \text { if } a \leq t \leq \sigma \leq b .\end{cases}
$$

Lemma 1 (cf. [13]). If $u \in W^{2, p}\left([a, b], \mathbb{R}^{n}\right), p \in[1, \infty]$, and $u(a)=$ $u(b)=\vartheta_{\mathbb{R}^{n}}$, then

$$
\begin{gathered}
u(t)=-\int_{a}^{b} K(t, \sigma) u^{\prime \prime}(\sigma) d \sigma, \\
u^{\prime}(t)=-\int_{a}^{b} \frac{\partial K(t, \sigma)}{\partial t} u^{\prime \prime}(\sigma) d \sigma .
\end{gathered}
$$

To simplify the notations, in the following Lemmas 2 and 3 we assume the indeterminate expressions, when $p=1$ or $p=\infty$, to be read as $\lim _{p \rightarrow 1^{+}}$ or $\lim _{p \rightarrow \infty}$ respectively.

Lemma 2 (cf. [13], Lemma 1.1). Let $p \in[1, \infty]$. Then, for every $t \in[a, b]$, we have

$$
\begin{aligned}
\|K(t, \cdot)\|_{L^{p}([a, b], \mathbb{R})} & \leq \frac{(b-a)^{1+1 / p}}{4(p+1)^{1 / p}} \\
\left\|\frac{\partial K(t, \sigma)}{\partial t}\right\|_{L^{p}([a, b], \mathbb{R})} & \leq \frac{(b-a)^{1 / p}}{(p+1)^{1 / p}} .
\end{aligned}
$$

In the following, $\|\cdot\|$ denotes a fixed norm on $\mathbb{R}^{n}$ and $d$ the metric induced by $\|\cdot\|$. 
Lemma 3. If $u \in W^{2, p}\left([a, b], \mathbb{R}^{n}\right), p \in[1, \infty]$, and $u(a)=u(b)=\vartheta_{\mathbb{R}^{n}}$, then, for every $t \in[a, b]$, we have

$$
\begin{aligned}
\|u(t)\| & \leq \frac{b-a}{4}\left[\frac{(b-a)(p-1)}{2 p-1}\right]^{1-1 / p}\left\|u^{\prime \prime}\right\|_{L^{p}\left([a, b], \mathbb{R}^{n}\right)}, \\
\left\|u^{\prime}(t)\right\| & \leq\left[\frac{(b-a)(p-1)}{2 p-1}\right]^{1-1 / p}\left\|u^{\prime \prime}\right\|_{L^{p}\left([a, b], \mathbb{R}^{n}\right)} .
\end{aligned}
$$

Moreover, for every $t, t^{*} \in[a, b]$ with $a \leq t<t^{*} \leq b$, we have

$$
\left\|u\left(t^{*}\right)-u(t)\right\| \leq\left[\frac{(b-a)(p-1)}{2 p-1}\right]^{1-1 / p}\left\|u^{\prime \prime}\right\|_{L^{p}\left([a, b], \mathbb{R}^{n}\right)}\left(t^{*}-t\right) .
$$

P r o of. By using (1), Hölder's inequality and (3), we obtain

$$
\begin{aligned}
\|u(t)\| & =\left\|\int_{a}^{b} K(t, \sigma) u^{\prime \prime}(\sigma) d \sigma\right\| \leq \int_{a}^{b}|K(t, \sigma)| \cdot\left\|u^{\prime \prime}(\sigma)\right\| d \sigma \\
& \leq\|K(t, \cdot)\|_{L^{p /(p-1)}([a, b], \mathbb{R})}\left\|u^{\prime \prime}\right\|_{L^{p}\left([a, b], \mathbb{R}^{n}\right)} \\
& \leq \frac{b-a}{4}\left[\frac{(b-a)(p-1)}{2 p-1}\right]^{1-1 / p}\left\|u^{\prime \prime}\right\|_{L^{p}\left([a, b], \mathbb{R}^{n}\right)}
\end{aligned}
$$

Similarly, by using (2), Hölder's inequality and (4), we obtain

$$
\begin{aligned}
\left\|u^{\prime}(t)\right\| & =\left\|\int_{a}^{b} \frac{\partial K(t, \sigma)}{\partial t} u^{\prime \prime}(\sigma) d \sigma\right\| \leq \int_{a}^{b}\left|\frac{\partial K(t, \sigma)}{\partial t}\right|\left\|u^{\prime \prime}(\sigma)\right\| d \sigma \\
& \leq\left\|\frac{\partial K(t, \cdot)}{\partial t}\right\|_{L^{p /(p-1)}([a, b], \mathbb{R})}\left\|u^{\prime \prime}\right\|_{L^{p}\left([a, b], \mathbb{R}^{n}\right)} \\
& \leq\left[\frac{(b-a)(p-1)}{2 p-1}\right]^{1-1 / p}\left\|u^{\prime \prime}\right\|_{L^{p}\left([a, b], \mathbb{R}^{n}\right) .}
\end{aligned}
$$

Finally, (7) is an immediate consequence of (6) and the weak form of the mean value theorem.

We recall that, given a set $L \in \mathcal{L}$, a point $t$ is a point of density for $L$ if

$$
\lim _{\eta \rightarrow 0^{+}} \frac{\mu(L \cap[t-\eta, t+\eta])}{2 \eta}=1 .
$$

The "density theorem" (cf., for instance, [16], p. 17) asserts that almost every point of a set $L \in \mathcal{L}$ is a point of density for $L$.

LEMmA 4. Let $G:[a, b] \times \mathbb{R}^{n} \times \mathbb{R}^{n} \rightarrow 2^{\mathbb{R}^{n}}$. Let $E \in \mathcal{L}, s \in[1, \infty]$, and $u \in W^{2, s}\left([a, b], \mathbb{R}^{n}\right)$ be such that $u^{\prime \prime}(t) \in G\left(t, u(t), u^{\prime}(t)\right)$ a.e. in $E$. Let $T$ be the set of all $t \in E$ such that: 
1) $u^{\prime \prime}(t) \in G\left(t, u(t), u^{\prime}(t)\right)$;

2) there exists a strictly decreasing sequence $\left(t_{j}\right)_{j}$ in $E$ such that

$$
t_{j} \stackrel{j}{\rightarrow} t, \quad u^{\prime \prime}\left(t_{j}\right) \stackrel{j}{\rightarrow} u^{\prime \prime}(t), \quad u^{\prime \prime}\left(t_{j}\right) \in G\left(t_{j}, u\left(t_{j}\right), u^{\prime}\left(t_{j}\right)\right) .
$$

Then $\mu(T)=\mu(E)$.

Proof. Let $T_{1}:=\left\{t \in E: u^{\prime \prime}(t) \in G\left(t, u(t), u^{\prime}(t)\right)\right\}$. By hypothesis, $\mu\left(T_{1}\right)=\mu(E)$.

Since $u^{\prime \prime} \in L^{s}\left([a, b], \mathbb{R}^{n}\right)$, in particular it satisfies the assumption of Lusin's theorem. Thus, for every $\varepsilon>0$ there exists $T_{\varepsilon} \subset[a, b]$ such that $\mu\left(T_{\varepsilon}\right)>b-a-\varepsilon$ and $u^{\prime \prime}{ }_{\mid T_{\varepsilon}}$ is continuous.

Put $T_{2}:=T_{1} \cap T_{\varepsilon}$. Then $\mu\left(T_{2}\right)=\mu\left(E \cap T_{\varepsilon}\right)>\mu(E)-\varepsilon, u^{\prime \prime}{ }_{\mid T_{2}}$ is continuous, and $u^{\prime \prime}(t) \in G\left(t, u(t), u^{\prime}(t)\right)$ for every $t \in T_{2}$.

Let $T_{3}$ be the set of all points of $T_{2}$ which are points of density for $T_{2}$. By the density theorem and the definition of point of density, we obtain $\mu\left(T_{3}\right)=\mu\left(T_{2}\right)>\mu(E)-\varepsilon$, and for every $t \in T_{3}$ there exists a strictly decreasing sequence $\left(t_{j}\right)_{j}$ in $T_{2}$, such that $t_{j} \stackrel{j}{\rightarrow} t$. Thus, $T_{3} \subset T$, so that $\mu(T) \geq \mu\left(T_{3}\right)>\mu(E)-\varepsilon$. Since $\varepsilon>0$ is arbitrary, the conclusion follows.

Lemma 5. Let $\left(A, \tau_{A}\right)$ be a topological space and $(Y, \varrho)$ a metric space. Let $F: A \rightarrow 2^{Y}$ be a lower semicontinuous multifunction, $m: A \rightarrow \mathbb{R}_{0}^{+}$ a lower semicontinuous function, and $y \in Y$. Then the multifunction $I_{y}$ : $A \rightarrow 2^{Y}$ defined by $I_{y}(t):=F(t) \cap B^{\circ}(y, m(t))$ is lower semicontinuous.

Proof. Let $\Omega$ be an open subset of $Y$ and $t_{0} \in I_{y}{ }^{-}(\Omega)$. Then there is $y_{0} \in F\left(t_{0}\right) \cap B^{\circ}\left(y, m\left(t_{0}\right)\right) \cap \Omega$. In particular, $\varrho\left(y_{0}, y\right)<m\left(t_{0}\right)$. Let $\delta>0$ be such that $\varrho\left(y_{0}, y\right)+\delta<m\left(t_{0}\right)$. Obviously, $y_{0} \in F\left(t_{0}\right) \cap B^{\circ}\left(y_{0}, \delta\right) \cap \Omega$. By the hypotheses on $F$ and $m$, there exists an open neighborhood $U$ of $t_{0}$ such that $F(t) \cap B^{\circ}\left(y_{0}, \delta\right) \cap \Omega \neq \emptyset$ and $\varrho\left(y_{0}, y\right)+\delta<m(t)$ for every $t \in U$. Then, for every $t \in U$, since $B^{\circ}\left(y_{0}, \delta\right) \subset B^{\circ}(y, m(t))$, we have $F(t) \cap B^{\circ}(y, m(t)) \cap \Omega$ $\neq \emptyset$.

Lemma 6. Let $\left(A, \mathcal{F}_{A}\right)$ be a measurable space, $\left(X, \tau_{X}\right)$ a second-countable topological space and $(Y, \varrho)$ a metric space in which bounded sets are relatively compact. Let $G: A \times X \rightarrow 2^{Y}$ be a multifuction, with non-empty values, such that:

$\left(\mathrm{i}_{1}\right) \bar{G}(t, \cdot)$ has closed graph for every $t \in A$;

$\left(\mathrm{i}_{2}\right)\left\{x \in X: G(\cdot, x)\right.$ is $\mathcal{F}_{A}$-measurable $\}$ is dense in $X$.

Then, for each $y \in Y$ and for each $B \subset X$ such that $\bar{B}=\overline{\operatorname{int}(B)} \neq \emptyset$, the extended real function $t \mapsto \sup _{x \in B} \varrho(y, G(t, x))$ is $\mathcal{F}_{A}$-measurable.

Proof. Let $\left\{B_{i}: i \in \mathbb{N}\right\}$ be a countable base for $\tau_{X}$. Put $\mathbb{N}_{B}:=\{i \in$ $\left.\mathbb{N}: B_{i} \cap B \neq \emptyset\right\}$. By $\left(\mathrm{i}_{2}\right)$, for each $i \in \mathbb{N}_{B}$ there exists $x_{i} \in B_{i} \cap \operatorname{int}(B)$ such 
that $\varrho\left(y, G\left(\cdot, x_{i}\right)\right)$ is $\mathcal{F}_{A}$-measurable. The countable set $D:=\left\{x_{i}: i \in \mathbb{N}_{B}\right\}$ is dense in $B$.

The extended real function $t \mapsto \sup _{i \in \mathbb{N}_{B}} \varrho\left(y, G\left(., x_{i}\right)\right)$ is $\mathcal{F}_{A^{-}}$-measurable; thus the conclusion follows if we prove that

$$
\sup _{x \in B} \varrho(y, G(t, x))=\sup _{i \in \mathbb{N}_{B}} \varrho\left(y, G\left(t, x_{i}\right)\right) \quad \text { for every } t \in A .
$$

Let $t \in A$. For every $x \in B$ and every $\varepsilon>0$, by using Proposition 1 of [15] and the density of $D$ in $B$, there exists $i_{0} \in \mathbb{N}_{B}$ such that

$$
\varrho(y, G(t, x))-\varepsilon<\varrho\left(y, G\left(t, x_{i_{0}}\right)\right) \leq \sup _{i \in \mathbb{N}_{B}} \varrho\left(y, G\left(t, x_{i}\right)\right) ;
$$

thus, $\varepsilon>0$ being arbitrary,

$$
\sup _{x \in B} \varrho(y, G(t, x)) \leq \sup _{i \in \mathbb{N}_{B}} \varrho\left(y, G\left(t, x_{i}\right)\right) .
$$

The opposite inequality is obvious.

3. Main result. Let $\|\cdot\|_{1},\|\cdot\|_{2}$ be two fixed norms on $\mathbb{R}^{n}$ (besides the already fixed norm $\|\cdot\|$, whose induced metric we have denoted by $d$ ). Define the norm $\|\cdot\|_{\mathbb{R}^{n} \times \mathbb{R}^{n}}$ on $\mathbb{R}^{n} \times \mathbb{R}^{n}$ by putting, for every $(x, z) \in \mathbb{R}^{n} \times \mathbb{R}^{n}$,

$$
\begin{aligned}
\|(x, z)\|_{\mathbb{R}^{n} \times \mathbb{R}^{n}}:=\max \left\{\max \left\{1, \frac{4}{b-a}\right\}\|x\|_{1}, \max \left\{1, \frac{b-a}{4}\right\}\|z\|_{2}\right\} \\
= \begin{cases}\max \left\{\frac{4}{b-a}\|x\|_{1},\|z\|_{2}\right\} \quad \text { if } b-a \leq 4, \\
\max \left\{\|x\|_{1}, \frac{b-a}{4}\|z\|_{2}\right\} \quad \text { if } b-a>4 .\end{cases}
\end{aligned}
$$

If $c_{1}, c_{2}$ are two positive constants such that

$$
\|x\|_{1} \leq c_{1}\|x\| \quad \text { and } \quad\|z\|_{2} \leq c_{2}\|z\| \quad \text { for all }(x, z) \in \mathbb{R}^{n} \times \mathbb{R}^{n},
$$

put $\gamma:=\gamma(p):=\max \left\{c_{1}, c_{2}\right\} \gamma^{\prime}$, where

$$
\gamma^{\prime}:=\gamma^{\prime}(p):= \begin{cases}\max \left\{1, \frac{b-a}{4}\right\}\left[\frac{(b-a)(p-1)}{2 p-1}\right]^{1-1 / p} & \text { if } 1<p<\infty, \\ \lim _{q \rightarrow 1^{+}} \gamma^{\prime}(q)=\max \left\{1, \frac{b-a}{4}\right\} & \text { if } p=1, \\ \lim _{q \rightarrow \infty} \gamma^{\prime}(q)=\max \left\{\frac{b-a}{2}, \frac{(b-a)^{2}}{8}\right\} & \text { if } p=\infty .\end{cases}
$$

Recall that, if $M>0$ is given and $\Gamma^{M}$ denotes the cone $\{(t, x, z) \in \mathbb{R} \times$ $\left.\mathbb{R}^{n} \times \mathbb{R}^{n}:\|(x, z)\|_{\mathbb{R}^{n} \times \mathbb{R}^{n}} \leq M t\right\}$, a function $h: E \rightarrow \mathbb{R}^{n}, E \subset \mathbb{R} \times \mathbb{R}^{n} \times \mathbb{R}^{n}$, is said to be $\Gamma^{M}$-continuous in $E$ if for every $(t, x, z) \in E$ and every $\varepsilon>0$ there exists $\delta>0$ such that $d\left(h\left(t^{*}, v, w\right), h(t, x, z)\right)<\varepsilon$ for every $\left(t^{*}, v, w\right) \in E$ such that $t<t^{*}<t+\delta$ and $\|(v, w)-(x, z)\|_{\mathbb{R}^{n} \times \mathbb{R}^{n}} \leq M\left(t^{*}-t\right)$. 
The following is our main result.

Theorem 1. Let $F:[a, b] \times \mathbb{R}^{n} \times \mathbb{R}^{n} \rightarrow 2^{\mathbb{R}^{n}}$. Suppose that there exist $p, s \in[1, \infty]$ with $p \leq s$, a non-negative function $m \in L^{s}([a, b], \mathbb{R})$, and $a$ positive number $r \geq\|m\|_{L^{p}([a, b], \mathbb{R})}$ such that

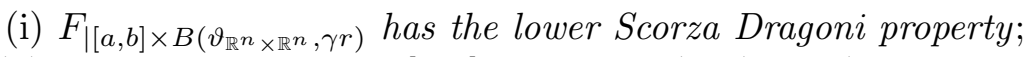

(ii) for almost every $t \in[a, b]$ and every $(x, z) \in B\left(\vartheta_{\mathbb{R}^{n} \times \mathbb{R}^{n}}, \gamma r\right)$, the set $F(t, x, z)$ is closed and $F(t, x, z) \cap B^{\circ}\left(\vartheta_{\mathbb{R}^{n}}, m(t)\right) \neq \emptyset$.

Then there exists a multifunction $G:[a, b] \times \mathbb{R}^{n} \times \mathbb{R}^{n} \rightarrow 2^{\mathbb{R}^{n}}$ with nonempty, closed and convex values such that

(j) $G(\cdot, x, z)$ is $\mathcal{L}$-measurable for every $(x, z) \in \mathbb{R}^{n} \times \mathbb{R}^{n}$,

(jj) $G(t, \cdot, \cdot)$ has closed graph for every $t \in[a, b]$,

(jjj) $G(t, x, z) \subset B\left(\vartheta_{\mathbb{R}^{n}}, m(t)\right)$ for every $(t, x, z) \in[a, b] \times \mathbb{R}^{n} \times \mathbb{R}^{n}$,

and every generalized solution $u$ of the problem $\left(\mathrm{P}_{G}\right)$ in $W^{2, s}\left([a, b], \mathbb{R}^{n}\right)$ is also a generalized solution of $\left(\mathrm{P}_{F}\right)$ and satisfies $\left\|u^{\prime \prime}(t)\right\| \leq m(t)$ a.e. in $[a, b]$.

Proof. Put $\left(X,\|\cdot\|_{X}\right):=\left(\mathbb{R}^{n} \times \mathbb{R}^{n},\|\cdot\|_{\mathbb{R}^{n} \times \mathbb{R}^{n}}\right),\left(Y,\|\cdot\|_{Y}\right):=\left(\mathbb{R}^{n},\|\cdot\|\right)$ and denote by $\vartheta_{X}$ and $\vartheta_{Y}$ the zero elements of $X$ and $Y$ respectively. Moreover, identify $(t,(x, z)) \in[a, b] \times X$ with $(t, x, z) \in[a, b] \times \mathbb{R}^{n} \times \mathbb{R}^{n}$ and $((x, z), y) \in$ $X \times Y$ with $(x, z, y) \in \mathbb{R}^{n} \times \mathbb{R}^{n} \times \mathbb{R}^{n}$.

Let $N$ be the set of all $t \in[a, b]$ such that, for some $(x, z) \in B\left(\vartheta_{X}, \gamma r\right)$, the set $F(t, x, z)$ is not closed or $F(t, x, z) \cap B^{\circ}\left(\vartheta_{Y}, m(t)\right)=\emptyset$. By (ii), we have $\mu(N)=0$.

Define $H:[a, b] \times X \rightarrow 2^{Y}$ by putting, for every $(t, x, z) \in[a, b] \times X$, $H(t, x, z):= \begin{cases}\overline{F(t, x, z) \cap B^{\circ}\left(\vartheta_{Y}, m(t)\right)} & \text { if }(t, x, z) \in([a, b] \backslash N) \times B\left(\vartheta_{X}, \gamma r\right), \\ B\left(\vartheta_{Y}, m(t)\right) & \text { if }(t, x, z) \notin([a, b] \backslash N) \times B\left(\vartheta_{X}, \gamma r\right) .\end{cases}$

We claim that $H$, which obviously has non-empty and closed values, has the lower Scorza Dragoni property.

For $\varepsilon>0$ fixed, let $T_{\varepsilon}$ be a compact subset of $[a, b] \backslash N$, with $\mu\left([a, b] \backslash T_{\varepsilon}\right)<$ $\varepsilon$, such that $F_{\mid T_{\varepsilon} \times B\left(\vartheta_{X}, \gamma r\right)}$ is lower semicontinuous and $m_{\mid T_{\varepsilon}}$ is continuous. Such a set exists since $F_{\mid[a, b] \times B\left(\vartheta_{X}, \gamma r\right)}$ has the lower Scorza Dragoni property, $m$ satisfies the assumption of Lusin's theorem, and $\mu(N)=0$.

Let $\Omega$ be an open subset of $Y$. Then

$$
\begin{aligned}
H_{\mid T_{\varepsilon} \times X}{ }^{-}(\Omega)= & \left\{(t, x, z) \in T_{\varepsilon} \times B\left(\vartheta_{X}, \gamma r\right):\right. \\
& \left.F(t, x, z) \cap B^{\circ}\left(\vartheta_{Y}, m(t)\right) \cap \Omega \neq \emptyset\right\} \\
& \cup\left[\left\{t \in T_{\varepsilon}: B^{\circ}\left(\vartheta_{Y}, m(t)\right) \cap \Omega \neq \emptyset\right\} \times\left(X \backslash B\left(\vartheta_{X}, \gamma r\right)\right)\right]
\end{aligned}
$$

and, as $(t, x, z) \mapsto F(t, x, z) \cap B^{\circ}\left(\vartheta_{Y}, m(t)\right)$ is lower semicontinuous in $T_{\varepsilon} \times$ $B\left(\vartheta_{X}, \gamma r\right)$ by Lemma 5 , and $m_{\mid T_{\varepsilon}}$ is continuous, it is simple to show that the last set is open in $T_{\varepsilon} \times X$. Thus $H$ has the lower Scorza Dragoni property. 
Now, by using a standard argument, we can find a sequence $\left(E_{i}\right)_{i}$, $i=0,1, \ldots$, of pairwise disjoint subsets of $[a, b]$ such that $[a, b]=\bigcup_{i=0}^{\infty} E_{i}$, $\mu\left(E_{0}\right)=0$, and, for every $i=1,2, \ldots, E_{i}$ is compact, $H_{\mid E_{i} \times X}$ is lower semicontinuous and $m_{\mid E_{i}}$ is continuous.

For each $i=1,2, \ldots$, put $m_{i}:=\max \left\{m(t): t \in E_{i}\right\}$ and choose $M_{i}>0$ such that (if $p=1$ or $p=\infty$, we assume the indeterminate expressions to be read as $\lim _{p \rightarrow 1^{+}}$or $\lim _{p \rightarrow \infty}$ respectively)

$$
\begin{array}{r}
M_{i}>\max \left\{c_{1}\left[\frac{(b-a)(p-1)}{2 p-1}\right]^{1-1 / p} r, \frac{4 c_{1}}{(b-a)^{1 / p}}\left(\frac{p-1}{2 p-1}\right)^{1-1 / p} r,\right. \\
\left.c_{2}\left(1+m_{i}\right), \frac{c_{2}(b-a)}{4}\left(1+m_{i}\right)\right\} .
\end{array}
$$

By Theorem 2.1 of [6], $H_{\mid E_{i} \times X}$ has a $\Gamma^{M_{i}}$-continuous selection $h_{i}$. Moreover, for $i=0$, by the axiom of choice, $H_{\mid E_{0} \times X}$ has a selection $h_{0}$. Define $h:[a, b] \times X \rightarrow Y$ by putting, for every $(t, x, z) \in[a, b] \times X$,

$$
h(t, x, z):=h_{i}(t, x, z) \quad \text { if } t \in E_{i}, i \in \mathbb{N} .
$$

The definition is correct, since the sets $E_{i}, i=0,1, \ldots$, are pairwise disjoint and $[a, b]=\bigcup_{i=0}^{\infty} E_{i}$.

Now, define $G:[a, b] \times X \rightarrow 2^{Y}$ by putting, for every $(t, x, z) \in[a, b] \times X$,

$$
G(t, x, z):=\bigcap_{\varepsilon>0} \overline{\operatorname{co}}\left\{h(t, v, w):\|(v, w)-(x, z)\|_{X}<\varepsilon\right\},
$$

where, as usual, $\overline{\text { co }}$ denotes the closed convex closure operator.

$G$, obviously, has non-empty, closed and convex values and satisfies (jjj).

Moreover, arguing for example as in [9], pp. 69-70, it can be easily proved that $G$ also satisfies $(\mathrm{j})$ and $(\mathrm{jj})$.

Now, let $u$ be a generalized solution of the problem $\left(\mathrm{P}_{G}\right)$ in $W^{2, s}([a, b], Y)$. Obviously, $\left\|u^{\prime \prime}(t)\right\| \leq m(t)$ a.e. in $[a, b]$. Thus, in particular,

$$
\left\|u^{\prime \prime}\right\|_{L^{p}([a, b], Y)} \leq r .
$$

Let us prove that $u^{\prime \prime}(t)=h\left(t, u(t), u^{\prime}(t)\right)$ a.e. in $[a, b]$ and $\left(u(t), u^{\prime}(t)\right) \in$ $B\left(\vartheta_{X}, \gamma r\right)$ a.e. in $[a, b]$, from which it follows that $u$ is a generalized solution of $\left(\mathrm{P}_{F}\right)$.

As the second assertion is an easy consequence of (5) and (6), we only prove the first. Since $b-a=\mu\left(\bigcup_{i=1}^{\infty} E_{i}\right)$, it is sufficient to prove that $u^{\prime \prime}(t)=$ $h_{i}\left(t, u(t), u^{\prime}(t)\right)$ a.e. in $E_{i}$ for every $i=1,2, \ldots$ Let $T$ be the set of all $t \in E_{i}$ such that:

1) $u^{\prime \prime}(t) \in G\left(t, u(t), u^{\prime}(t)\right)$;

2) there exists a strictly decreasing sequence $\left(t_{j}\right)_{j}$ in $E_{i}$ such that 


$$
t_{j} \stackrel{j}{\rightarrow} t, \quad u^{\prime \prime}\left(t_{j}\right) \stackrel{j}{\rightarrow} u^{\prime \prime}(t), \quad u^{\prime \prime}\left(t_{j}\right) \in G\left(t_{j}, u\left(t_{j}\right), u^{\prime}\left(t_{j}\right)\right) .
$$

Then, by Lemma $4, \mu(T)=\mu\left(E_{i}\right)$.

We prove that, for every $t \in T, u^{\prime \prime}(t)=h_{i}\left(t, u(t), u^{\prime}(t)\right)$.

Fix $\varepsilon>0$. By the $\Gamma^{M_{i}}$-continuity of $h_{\mid E_{i}}$ in $\left(t, u(t), u^{\prime}(t)\right)$, there exists $\delta>0$ such that, for every $\left(t^{*}, v, w\right) \in E_{i} \times X$ with $t<t^{*}<t+\delta$ and $\left\|(v, w)-\left(u(t), u^{\prime}(t)\right)\right\|_{X} \leq M_{i}\left(t^{*}-t\right)$, we have $d\left(h\left(t^{*}, v, w\right), h\left(t, u(t), u^{\prime}(t)\right)\right)<$ $\varepsilon / 2$.

Since $t_{j} \stackrel{j}{\rightarrow} t$, there exists $j_{0} \in \mathbb{N}$ such that, for every $j \in \mathbb{N}$ with $j>j_{0}$, we have

$$
t<t_{j}<t+\delta, \quad d\left(u^{\prime \prime}\left(t_{j}\right), u^{\prime \prime}(t)\right)<\varepsilon / 2, \quad u^{\prime \prime}\left(t_{j}\right) \in G\left(t_{j}, u\left(t_{j}\right), u^{\prime}\left(t_{j}\right)\right)
$$

and

$$
\left\|\frac{u^{\prime}\left(t_{j}\right)-u^{\prime}(t)}{t_{j}-t}-u^{\prime \prime}(t)\right\|<1
$$

Then, for every $j \in \mathbb{N}$ with $j>j_{0}$, we obtain

$$
\begin{aligned}
\left\|u^{\prime}\left(t_{j}\right)-u^{\prime}(t)\right\| & =\left\|\frac{u^{\prime}\left(t_{j}\right)-u^{\prime}(t)}{t_{j}-t}\right\|\left(t_{j}-t\right) \\
& \leq\left(\left\|\frac{u^{\prime}\left(t_{j}\right)-u^{\prime}(t)}{t_{j}-t}-u^{\prime \prime}(t)\right\|+\left\|u^{\prime \prime}(t)\right\|\right)\left(t_{j}-t\right) \\
& \leq\left(1+m_{i}\right)\left(t_{j}-t\right) .
\end{aligned}
$$

Taking into account (7)-(10), it is simple to verify that

$$
\left\|\left(u\left(t_{j}\right), u^{\prime}\left(t_{j}\right)\right)-\left(u(t), u^{\prime}(t)\right)\right\|_{X}<M_{i}\left(t_{j}-t\right),
$$

hence

$$
G\left(t_{j}, u\left(t_{j}\right), u^{\prime}\left(t_{j}\right)\right) \subset B\left(h_{i}\left(t, u(t), u^{\prime}(t)\right), \varepsilon / 2\right),
$$

and thus

$$
d\left(u^{\prime \prime}\left(t_{j}\right), h_{i}\left(t, u(t), u^{\prime}(t)\right)\right) \leq \varepsilon / 2 .
$$

Therefore, we obtain

$d\left(u^{\prime \prime}(t), h_{i}\left(t, u(t), u^{\prime}(t)\right)\right) \leq d\left(u^{\prime \prime}(t), u^{\prime \prime}\left(t_{j}\right)\right)+d\left(u^{\prime \prime}\left(t_{j}\right), h_{i}\left(t, u(t), u^{\prime}(t)\right)\right)<\varepsilon$, from which $u^{\prime \prime}(t)=h\left(t, u(t), u^{\prime}(t)\right)$ follows, since $\varepsilon$ is arbitrary.

REMARK 1. In Theorem 1 (and in Theorem 2 below), the hypothesis (ii) can be replaced by

(ii) for almost every $t \in[a, b]$ and every $(x, z) \in B\left(\vartheta_{\mathbb{R}^{n} \times \mathbb{R}^{n}}, \gamma r\right)$, the set $F(t, x, z)$ is closed and $\emptyset \neq F(t, x, z) \subset B\left(\vartheta_{\mathbb{R}^{n}}, m(t)\right)$.

The proof differs from that of Theorem 1 only in the definition of $H$. Under (ii)' one can use $H:[a, b] \times \mathbb{R}^{n} \times \mathbb{R}^{n} \rightarrow 2^{\mathbb{R}^{n}}$ defined by putting, for 
every $(t, x, z) \in[a, b] \times \mathbb{R}^{n} \times \mathbb{R}^{n}$,

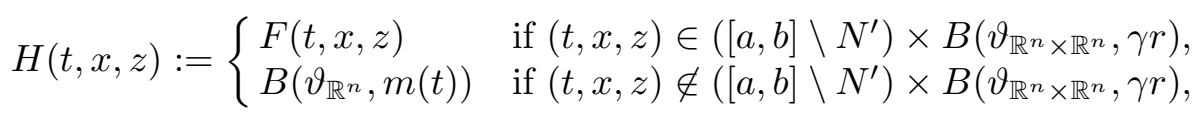

where $N^{\prime}$ is the set of all $t \in[a, b]$ such that, for some $(x, z) \in B\left(\vartheta_{\mathbb{R}^{n} \times \mathbb{R}^{n}}, \gamma r\right)$, $F(t, x, z)$ is empty or not closed or $F(t, x, z) \not \subset B\left(\vartheta_{\mathbb{R}^{n}}, m(t)\right)$.

It is not difficult to show that $H$ has non-empty and closed values and has the lower Scorza Dragoni property.

REMARK 2. It is well known that $F_{\mid[a, b] \times B\left(\vartheta_{\mathbb{R}^{n}} \times \mathbb{R}^{n}, \gamma r\right)}$ has the lower Scorza

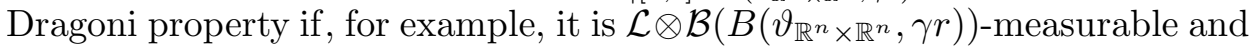
lower semicontinuous in $(x, z)$, or if it is $\mathcal{L}$-measurable in $t$ and continuous in $(x, z)$.

There is extensive literature on this topic (see, for example, [2]-[4], [7], [12] and the recent survey [1]).

Also mixed properties of the multifunction guarantee the lower Scorza Dragoni property. We mention here Theorem 2 of [4].

When the multifunction $F$ is weakly integrably bounded by a function $m^{*} \in L^{s}([a, b], \mathbb{R})$, the following result is a corollary of Theorem 1 .

Corollary 1. Let $F:[a, b] \times \mathbb{R}^{n} \times \mathbb{R}^{n} \rightarrow 2^{\mathbb{R}^{n}}$ have the lower Scorza Dragoni property. Suppose that there exist $s \in[1, \infty]$ and a non-negative function $m^{*} \in L^{s}([a, b], \mathbb{R})$ such that

(ii)" for almost every $t \in[a, b]$ and every $(x, z) \in \mathbb{R}^{n} \times \mathbb{R}^{n}$, the set $F(t, x, z)$ is closed and $F(t, x, z) \cap B\left(\vartheta_{\mathbb{R}^{n}}, m^{*}(t)\right) \neq \emptyset$.

Then for each $\lambda>0$ there exists a multifunction $G_{\lambda}:[a, b] \times \mathbb{R}^{n} \times \mathbb{R}^{n} \rightarrow$ $2^{\mathbb{R}^{n}}$ with non-empty, closed and convex values such that

(j) $G_{\lambda}(\cdot, x, z)$ is $\mathcal{L}$-measurable for every $(x, z) \in \mathbb{R}^{n} \times \mathbb{R}^{n}$,

(jj) $G_{\lambda}(t, \cdot, \cdot)$ has closed graph for every $t \in[a, b]$,

(jjj) $G_{\lambda}(t, x, z) \subset B\left(\vartheta_{\mathbb{R}^{n}}, m^{*}(t)+\lambda\right)$ for every $(t, x, z) \in[a, b] \times \mathbb{R}^{n} \times \mathbb{R}^{n}$, and every generalized solution $u$ of the problem $\left(\mathrm{P}_{G_{\lambda}}\right)$ in $W^{2, s}\left([a, b], \mathbb{R}^{n}\right)$ is also a generalized solution of $\left(\mathrm{P}_{F}\right)$ and satisfies $\left\|u^{\prime \prime}(t)\right\| \leq m^{*}(t)+\lambda$ a.e. in $[a, b]$.

Proof. Fix $\lambda>0$. Then $F$ satisfies (ii) of Theorem 1 with $m:=m^{*}+\lambda$, $p=s$, and $r:=\left\|m^{*}+\lambda\right\|_{L^{p}([a, b], \mathbb{R})}$.

4. Existence. In this section $\|\cdot\|, d,\|\cdot\|_{\mathbb{R}^{n} \times \mathbb{R}^{n}}$ and $\gamma$ are as at the beginning of Section 3.

The following existence theorem follows at once from Theorem 1, Lemma 6 and Theorem 2.1 of [13]. 
THEOREM 2. Let $F$ be a multifunction as in Theorem 1 (in which (ii) or (ii)' can be used; see Remark 1$)$. Then the problem $\left(\mathrm{P}_{F}\right)$ has at least one generalized solution $u \in W^{2, s}\left([a, b], \mathbb{R}^{n}\right)$ such that $\left\|u^{\prime \prime}(t)\right\| \leq m(t)$ a.e. in $[a, b]$.

Proof. Let $G$ be the multifunction whose existence has been stated in Theorem 1, and $r \geq\|m\|_{L^{p}([a, b], \mathbb{R})}$ a positive number. By Lemma 6 , $t \mapsto \sup \left\{d\left(\vartheta_{\mathbb{R}^{n}}, G(t, x, z)\right):(x, z) \in B\left(\vartheta_{\mathbb{R}^{n}} \times \mathbb{R}^{n}, \gamma r\right)\right\}$ is $\mathcal{L}$-measurable. Thus, by (ii) and as $d\left(\vartheta_{\mathbb{R}^{n}}, G(t, x, z)\right) \leq m(t)$ for every $(t, x, z) \in[a, b] \times \mathbb{R}^{n} \times \mathbb{R}^{n}$, it follows that $t \mapsto \sup \left\{d\left(\vartheta_{\mathbb{R}^{n}}, G(t, x, z)\right):(x, z) \in B\left(\vartheta_{\mathbb{R}^{n} \times \mathbb{R}^{n}}, \gamma r\right)\right\}$ belongs to $L^{s}([a, b], \mathbb{R})$ and its norm in $L^{p}([a, b], \mathbb{R})$ is less than or equal to $r$.

Hence, we can use Theorem 2.1 of [13] to obtain a generalized solution $u$ of $\left(\mathrm{P}_{G}\right)$ in $W^{2, s}\left([a, b], \mathbb{R}^{n}\right)$, which, by Theorem 1 , is also a generalized solution of $\left(\mathrm{P}_{F}\right)$ such that $\left\|u^{\prime \prime}(t)\right\| \leq m(t)$ a.e. in $[a, b]$.

Corollary 2. Let $F$ be as in Corollary 1. Then for each $\lambda>0$ the problem $\left(\mathrm{P}_{F}\right)$ has at least one generalized solution $u_{\lambda} \in W^{2, s}\left([a, b], \mathbb{R}^{n}\right)$ such that $\left\|u_{\lambda}^{\prime \prime}(t)\right\| \leq m^{*}(t)+\lambda$ a.e. in $[a, b]$.

REMARK 3. Theorem 2 and Corollary 2 both improve Theorem 3 of [8], in which $F$ has non-empty and compact values and is measurable in $t$, Hausdorff continuous in $(x, z)$ and integrably bounded.

The following existence theorem is a consequence of our Theorem 2 and Theorem 11 of [18].

THEOREM 3. Let I be a non-empty subset of $\mathbb{R}$ and $F: I \rightarrow 2^{\mathbb{R}^{n}}$ a multifunction with non-empty and closed values such that:

$(\alpha) \operatorname{gr}(F)$ is connected and locally connected;

$(\alpha \alpha)$ for every open set $\Omega \subset \mathbb{R}^{n}$, the set $F^{-}(\Omega) \cap \operatorname{int}(I)$ has no isolated points.

Moreover, let $G:[a, b] \times \mathbb{R}^{n} \times \mathbb{R}^{n} \rightarrow 2^{\mathbb{R}}$ be a multifunction with non-empty values, $p, s \in[1, \infty]$, with $p \leq s, m \in L^{s}([a, b], \mathbb{R})$ a non-negative function, and $r \geq\|m\|_{L^{p}([a, b], \mathbb{R})}$ a positive number such that:

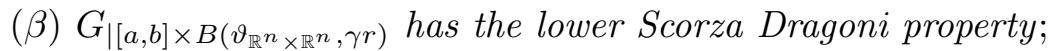

$(\beta \beta)$ for almost every $t \in[a, b]$ and every $(x, z) \in B\left(\vartheta_{\mathbb{R}^{n} \times \mathbb{R}^{n}}, \gamma r\right)$, the set $G(t, x, z)$ is a compact subset of $I$ and $G(t, x, z) \cap F^{+}\left(B^{\circ}\left(\vartheta_{\mathbb{R}^{n}}, m(t)\right)\right) \neq \emptyset$.

Then there exists a generalized solution $u \in W^{2, s}\left([a, b], \mathbb{R}^{n}\right)$ of the problem

$\left(\mathrm{P}_{F \circ G}\right) \quad\left\{\begin{array}{l}u^{\prime \prime} \in F\left(G\left(t, u, u^{\prime}\right)\right), \\ u(a)=u(b)=\vartheta_{\mathbb{R}^{n}},\end{array}\right.$

such that $\left\|u^{\prime \prime}(t)\right\| \leq m(t)$ a.e. in $[a, b]$. 
Proof. Thanks to our assumptions on $F$, we can apply Theorem 11 of [18]. Hence, there exist $\Phi_{1}, \Phi_{2}: I \rightarrow 2^{\mathbb{R}^{n}}$ such that $\Phi_{1}$ is lower semicontinuous, $\Phi_{2}$ is upper semicontinuous with compact values, and $\emptyset \neq \Phi_{1}(v) \subset$ $\Phi_{2}(v) \subset F(v)$ for every $v \in I$.

Let $N_{0} \subset[a, b]$ with $\mu\left(N_{0}\right)=0$ be such that, for every $(t, x, z) \in$

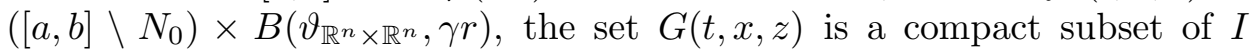
and $G(t, x, z) \cap F^{+}\left(B^{\circ}\left(\vartheta_{\mathbb{R}^{n}}, m(t)\right)\right) \neq \emptyset$.

For every $(t, x, z) \in[a, b] \times \mathbb{R}^{n} \times \mathbb{R}^{n}$, put

$$
\Gamma(t, x, z):= \begin{cases}\overline{\Phi_{1}(G(t, x, z))} & \text { if }(t, x, z) \in\left([a, b] \backslash N_{0}\right) \times B\left(\vartheta_{\mathbb{R}^{n} \times \mathbb{R}^{n}}, \gamma r\right), \\ \mathbb{R}^{n} & \text { if }(t, x, z) \notin\left([a, b] \backslash N_{0}\right) \times B\left(\vartheta_{\mathbb{R}^{n} \times \mathbb{R}^{n}}, \gamma r\right) .\end{cases}
$$

Obviously, the values of $\Gamma$ are non-empty and closed and it is simple to see that $\Gamma$ has the lower Scorza Dragoni property.

Moreover, for every $t \in\left([a, b] \backslash N_{0}\right) \times B\left(\vartheta_{\mathbb{R}^{n}} \times \mathbb{R}^{n}, \gamma r\right)$, we have

$$
\Gamma(t, x, z) \cap B^{\circ}\left(\vartheta_{\mathbb{R}^{n}}, m(t)\right) \neq \emptyset .
$$

In fact, we have

$$
G(t, x, z) \cap \Phi_{1}^{+}\left(B^{\circ}\left(\vartheta_{\mathbb{R}^{n}}, m(t)\right)\right) \neq \emptyset,
$$

hence

$$
G(t, x, z) \cap \Phi_{1}^{-}\left(B^{\circ}\left(\vartheta_{\mathbb{R}^{n}}, m(t)\right)\right) \neq \emptyset,
$$

and then

$$
\Phi_{1}(G(t, x, z)) \cap\left(B^{\circ}\left(\vartheta_{\mathbb{R}^{n}}, m(t)\right)\right) \neq \emptyset,
$$

which is equivalent to

$$
\overline{\Phi_{1}(G(t, x, z))} \cap\left(B^{\circ}\left(\vartheta_{\mathbb{R}^{n}}, m(t)\right)\right) \neq \emptyset .
$$

By Theorem 2 (with the hypothesis (ii)) applied to $\Gamma$, there exists $u \in$ $W^{2, s}\left([a, b], \mathbb{R}^{n}\right)$ such that

$$
\left\{\begin{array}{l}
u^{\prime \prime}(t) \in \Gamma\left(t, u(t), u^{\prime}(t)\right) \quad \text { for a.e. } t \in[a, b] \\
u(a)=u(b)=\vartheta_{\mathbb{R}^{n}}
\end{array}\right.
$$

and $\left\|u^{\prime \prime}(t)\right\| \leq m(t)$ a.e. in $[a, b]$. The function $u$ is our solution.

In fact, by (5) and (6), we have $\left(u(t), u^{\prime}(t)\right) \in B\left(\vartheta_{\mathbb{R}^{n} \times \mathbb{R}^{n}}, \gamma r\right)$ for every $t \in[a, b]$. Then

$$
\left\{\begin{array}{l}
u^{\prime \prime}(t) \in \overline{\Phi_{1}\left(G\left(t, u(t), u^{\prime}(t)\right)\right)} \quad \text { for a.e. } t \in[a, b] \\
u(a)=u(b)=\vartheta_{\mathbb{R}^{n}}
\end{array}\right.
$$

and, for almost every $t \in[a, b], G\left(t, u(t), u^{\prime}(t)\right)$ is a compact subset of $I$, hence, by Theorem 2.1 of [11], $\Phi_{2}\left(G\left(t, u(t), u^{\prime}(t)\right)\right)$ is compact.

Thus, for almost every $t \in[a, b]$, we have

$$
\Phi_{1}\left(G\left(t, u(t), u^{\prime}(t)\right)\right) \subset \Phi_{2}\left(G\left(t, u(t), u^{\prime}(t)\right)\right)=\overline{\Phi_{2}\left(G\left(t, u(t), u^{\prime}(t)\right)\right)},
$$


therefore

$$
\overline{\Phi_{1}\left(G\left(t, u(t), u^{\prime}(t)\right)\right)} \subset F\left(G\left(t, u(t), u^{\prime}(t)\right)\right),
$$

from which the conclusion follows.

REMARK 4 . In Theorem 3 the hypothesis $(\beta \beta)$ can be replaced by

$(\beta \beta)^{\prime}$ for almost every $t \in[a, b]$ and every $(x, z) \in B\left(\vartheta_{\mathbb{R}^{n} \times \mathbb{R}^{n}}, \gamma r\right)$, the set $G(t, x, z)$ is a compact subset of $I$ and $F(G(t, x, z)) \subset B\left(\vartheta_{\mathbb{R}^{n}}, m(t)\right)$.

In fact the multifunction $\Gamma$ defined in the proof of Theorem 3 satisfies the assumptions of Theorem 2 with (ii)' in place of (ii).

REMARK 5. An existence result for the Cauchy problem for a first order differential inclusion with right-hand side of the type $F \circ G$ has recently been given in [5].

5. Applications. In this section, we give some applications of our results to the existence of solutions for a boundary value problem for second-order implicit equations. $\|\cdot\|,\|\cdot\|_{\mathbb{R}^{n} \times \mathbb{R}^{n}}$ and $\gamma$ are as at the beginning of Section 3 .

THEOREM 4. Let $Y$ be a non-empty, connected and locally connected subset of $\mathbb{R}^{n}$ and $f:[a, b] \times \mathbb{R}^{n} \times \mathbb{R}^{n} \times Y \rightarrow \mathbb{R}$. Assume that there exist $p, s \in[1, \infty]$, with $p \leq s$, a non-negative function $m \in L^{s}([a, b], \mathbb{R})$, and $a$ positive number $r \geq\|m\|_{L^{p}([a, b], \mathbb{R})}$, such that:

(k) for almost every $t \in[a, b]$ and every $(x, z) \in B\left(\vartheta_{\mathbb{R}^{n} \times \mathbb{R}^{n}}, \gamma r\right)$, the function $f(t, x, z, \cdot)$ is continuous, $0 \in \operatorname{int}\left(f\left(t, x, z, Y \cap B^{\circ}\left(\vartheta_{\mathbb{R}^{n}}, m(t)\right)\right)\right)$, and $\{y \in Y: f(t, x, z, y)=0\}$ has empty interior in $Y$;

(kk) for every $\varepsilon>0$ there exists a compact set $T_{\varepsilon} \subset[a, b]$ with $\mu\left([a, b] \backslash T_{\varepsilon}\right)<\varepsilon$ and a set $D_{\varepsilon} \subset Y \times Y$ with $\bar{D}_{\varepsilon} \supset Y \times Y$ such that, for every $\left(y^{\prime}, y^{\prime \prime}\right) \in D_{\varepsilon}$, the set $\left\{(t, x, z) \in T_{\varepsilon} \times B\left(\vartheta_{\mathbb{R}^{n} \times \mathbb{R}^{n}}, \gamma r\right): f\left(t, x, z, y^{\prime}\right)<\right.$ $\left.0<f\left(t, x, z, y^{\prime \prime}\right)\right\}$ is open in $T_{\varepsilon} \times B\left(\vartheta_{\mathbb{R}^{n} \times \mathbb{R}^{n}}, \gamma r\right)$;

(kkk) for almost every $t \in[a, b]$, the set $Y \cap B^{\circ}\left(\vartheta_{\mathbb{R}^{n}}, m(t)\right)$ is connected.

Then the problem

$$
\left\{\begin{array}{l}
f\left(t, u, u^{\prime}, u^{\prime \prime}\right)=0 \\
u(a)=u(b)=\vartheta_{\mathbb{R}^{n}}
\end{array}\right.
$$

has at least one generalized solution $u$ in $W^{2, s}\left([a, b], \mathbb{R}^{n}\right)$ such that $\left\|u^{\prime \prime}(t)\right\| \leq$ $m(t)$ a.e. in $[a, b]$.

Proof. Define $Q:[a, b] \times B\left(\vartheta_{\mathbb{R}^{n} \times \mathbb{R}^{n}}, \gamma r\right) \rightarrow 2^{Y}$ by putting, for every $(t, x, z) \in[a, b] \times B\left(\vartheta_{\mathbb{R}^{n}} \times \mathbb{R}^{n}, \gamma r\right)$,

$Q(t, x, z)$

$:=\{y \in Y: f(t, x, z, y)=0, y$ is not a local extremum point for $f(t, x, z, \cdot)\}$. 
For every $\varepsilon>0$, let $T_{\varepsilon}$ be a compact subset of $[a, b]$ as in (kk) such that, for every $(t, x, z) \in T_{\varepsilon} \times B\left(\vartheta_{\mathbb{R}^{n} \times \mathbb{R}^{n}}, \gamma r\right)$, the function $f(t, x, z, \cdot)$ is continuous, $0 \in \operatorname{int}\left(f\left(t, x, z, Y \cap B^{\circ}\left(\vartheta_{\mathbb{R}^{n}}, m(t)\right)\right)\right)$ and the set $\{y \in Y: f(t, x, z, y)=0\}$ has empty interior in $Y$.

By Théorème 1.1 of [17], $Q_{\mid T_{\varepsilon} \times B\left(\vartheta_{\left.\mathbb{R}^{n} \times \mathbb{R}^{n}, \gamma r\right)}\right.}$ (has non-empty and closed values (in $Y$ ) and) is lower semicontinuous. Thus $Q$ has the lower Scorza Dragoni property.

We claim that, for almost every $t \in[a, b]$ and every $(x, z) \in B\left(\vartheta_{\mathbb{R}^{n}} \times \mathbb{R}^{n}, \gamma r\right)$, the set $Q(t, x, z)$ is closed and $Q(t, x, z) \cap B^{\circ}\left(\vartheta_{\mathbb{R}^{n}}, m(t)\right) \neq \emptyset$.

Let $T$ be the set of all $t \in[a, b]$ such that $Y \cap B^{\circ}\left(\vartheta_{\mathbb{R}^{n}}, m(t)\right)$ is connected and such that, for every $(x, z) \in B\left(\vartheta_{\mathbb{R}^{n} \times \mathbb{R}^{n}}, \gamma r\right)$, the function $f(t, x, z, \cdot)$ is continuous, $0 \in \operatorname{int}\left(f\left(t, x, z, Y \cap B^{\circ}\left(\vartheta_{\mathbb{R}^{n}}, m(t)\right)\right)\right)$, and $\{y \in Y: f(t, x, z, y)$ $=0\}$ has empty interior in $Y$ and, thus, also in $Y \cap B^{\circ}\left(\vartheta_{\mathbb{R}^{n}}, m(t)\right)$. Clearly, $\mu(T)=b-a$.

Let $(t, x, z) \in T \times B\left(\vartheta_{\mathbb{R}_{n} \times \mathbb{R}^{n}}, \gamma r\right)$.

If $\bar{y} \in \overline{Q(t, x, z)}$, then $f(t, x, z, \bar{y})=0$ since $f(t, x, z, \cdot)$ is continuous; moreover, for every open neighborhood $\Omega$ of $\bar{y}$, there is $y^{*} \in Q(t, x, z) \cap \Omega$. Thus, since $y^{*}$ is not a local extremum point for $f(t, x, z, \cdot)$ and $f(t, x, z, \bar{y})=$ $f\left(t, x, z, y^{*}\right)=0$, also $\bar{y}$ is not a local extremum point for $f(t, x, z, \cdot)$, that is, $\bar{y} \in Q(t, x, z)$. Hence $Q(t, x, z)$ is closed.

Let $y \in Y \cap B^{\circ}\left(\vartheta_{\mathbb{R}^{n}}, m(t)\right)$ be such that $f(t, x, z, y)=0$. If $y$ is not a local extremum point for $f(t, x, z, \cdot)$, then $y \in Q(t, x, z) \cap B^{\circ}\left(\vartheta_{\mathbb{R}^{n}}, m(t)\right)$. If $y$ is a local extremum point for $f(t, x, z, \cdot)$, then, by Lemma 3.1 of [19], there exists another point $y^{*} \in Y \cap B^{\circ}\left(\vartheta_{\mathbb{R}^{n}}, m(t)\right)$ such that $f\left(t, x, z, y^{*}\right)=0$ and $y^{*}$ is not a local extremum point for $f(t, x, z, \cdot)$, that is, $y^{*} \in Q(t, x, z) \cap$ $B^{\circ}\left(\vartheta_{\mathbb{R}^{n}}, m(t)\right)$. Hence, $Q(t, x, z) \cap B^{\circ}\left(\vartheta_{\mathbb{R}^{n}}, m(t)\right)$ is non-empty and the claim is proved.

Finally, define $F:[a, b] \times \mathbb{R}^{n} \times \mathbb{R}^{n} \rightarrow 2^{\mathbb{R}^{n}}$ by putting, for every $(t, x, z) \in$ $[a, b] \times \mathbb{R}^{n} \times \mathbb{R}^{n}$,

$$
F(t, x, z):= \begin{cases}Q(t, x, z) & \text { if }(x, z) \in B\left(\vartheta_{\mathbb{R}^{n} \times \mathbb{R}^{n}}, \gamma r\right), \\ \mathbb{R}^{n} & \text { if }(x, z) \notin B\left(\vartheta_{\mathbb{R}^{n} \times \mathbb{R}^{n}}, \gamma r\right) .\end{cases}
$$

$F$ satisfies the hypotheses of Theorem 2. Thus, $\left(\mathrm{P}_{F}\right)$ has at least one generalized solution $u$ in $W^{2, s}\left([a, b], \mathbb{R}^{n}\right)$ such that $\left\|u^{\prime \prime}(t)\right\| \leq m(t)$ a.e. in $[a, b]$. Taking into account (5), (6), it is simple to show that $\left(u(t), u^{\prime}(t)\right) \in$ $B\left(\vartheta_{\mathbb{R}^{n} \times \mathbb{R}^{n}}, \gamma r\right)$ for every $t \in[a, b]$, so that $u^{\prime \prime}(t) \in Q\left(t, u(t), u^{\prime}(t)\right)$ a.e. in $[a, b]$, that is, $f\left(t, u(t), u^{\prime}(t), u^{\prime \prime}(t)\right)=0$ a.e. in $[a, b]$.

In the following Theorem 5 , we put $p=s=\infty$ and $\gamma:=\gamma(\infty)$.

THEOREM 5. Let $Y$ be a non-empty, connected and locally connected subset of $\mathbb{R}^{n}$, and $f:[a, b] \times \mathbb{R}^{n} \times \mathbb{R}^{n} \times Y \rightarrow \mathbb{R}$. Assume that there exists $r>0$ such that $Y \subset B\left(\vartheta_{\mathbb{R}^{n}}, r\right)$ and: 
$(\mathrm{k})^{\prime}$ for almost every $t \in[a, b]$ and every $(x, z) \in B\left(\vartheta_{\mathbb{R}^{n} \times \mathbb{R}^{n}}, \gamma r\right)$, the function $f(t, x, z, \cdot)$ is continuous, $0 \in \operatorname{int}(f(t, x, z, Y))$, and $\{y \in Y$ : $f(t, x, z, y)=0\}$ has empty interior in $Y$;

(kk) for every $\varepsilon>0$ there exists a compact set $T_{\varepsilon} \subset[a, b]$ with $\mu\left([a, b] \backslash T_{\varepsilon}\right)$ $<\varepsilon$ and a set $D_{\varepsilon} \subset Y \times Y$ with $\bar{D}_{\varepsilon} \supset Y \times Y$ such that, for every $\left(y^{\prime}, y^{\prime \prime}\right) \in$ $D_{\varepsilon}$, the set $\left\{(t, x, z) \in T_{\varepsilon} \times B\left(\vartheta_{\mathbb{R}^{n} \times \mathbb{R}^{n}}, \gamma r\right): f\left(t, x, z, y^{\prime}\right)<0<f\left(t, x, z, y^{\prime \prime}\right)\right\}$ is open in $T_{\varepsilon} \times B\left(\vartheta_{\mathbb{R}^{n} \times \mathbb{R}^{n}}, \gamma r\right)$.

Then the problem $\left(\mathrm{P}_{f}^{i}\right)$ has at least one generalized solution $u$ in the space $W^{2, \infty}\left([a, b], \mathbb{R}^{n}\right)$ such that $\left\|u^{\prime \prime}(t)\right\| \leq r$ a.e. in $[a, b]$.

Proof. Put $m(t):=r$ for every $t \in[a, b]$, define $Q$ and $F$ as in Theorem 4 , and use Theorem 2 with (ii)' instead of (ii).

Remark 6. In Theorems 4 and 5, the hypothesis (kk) is satisfied, in par-

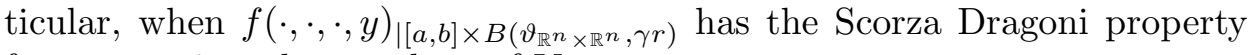
for every $y$ in a dense subset of $Y$.

We observe that the hypothesis (kk) in Theorem 5 could be substituted with (ii) and (iii) of Theorem 2.1 of [14]; in fact, with these hypotheses the multifunction $Q$ is $\mathcal{L} \otimes \mathcal{B}\left(B\left(\vartheta_{\mathbb{R}^{n} \times \mathbb{R}^{n}}, \gamma r\right)\right)$-measurable and lower semicontinuous in $(x, z)$, thus (see Remark 2 ) it has the lower Scorza Dragoni property. In any case, in Theorem 2.1 of [14] the set $Y$ is also compact.

Theorem 6. Let $Y$ be a non-empty subset of $\mathbb{R}^{n}$ and $f: Y \rightarrow \mathbb{R}$ such that:

$(\alpha) \operatorname{gr}(f)$ is connected and locally connected;

$(\alpha \alpha)^{\prime}$ for every $v \in \operatorname{int}(f(Y))$, the set $f^{-1}(v)$ has empty interior in $Y$;

$(\alpha \alpha \alpha)$ for every $v \in f(Y)$, the set $f^{-1}(v)$ is closed in $\mathbb{R}^{n}$.

Moreover, let $g:[a, b] \times \mathbb{R}^{n} \times \mathbb{R}^{n} \rightarrow \mathbb{R}, p, s \in[1, \infty]$, with $p \leq s$, $m \in L^{s}([a, b], \mathbb{R})$ a non-negative function, and $r \geq\|m\|_{L^{p}([a, b], \mathbb{R})}$ a positive number such that:

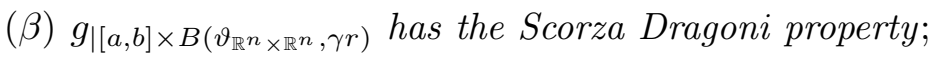

$(\beta \beta)^{\prime}$ for almost every $t \in[a, b]$ and every $(x, z) \in B\left(\vartheta_{\mathbb{R}^{n} \times \mathbb{R}^{n}}, \gamma r\right)$, we have $\emptyset \neq f^{-1}(g(t, x, z)) \subset B\left(\vartheta_{\mathbb{R}^{n}}, m(t)\right)$.

Then the problem

$$
\left\{\begin{array}{l}
f\left(u^{\prime \prime}\right)=g\left(t, u, u^{\prime}\right) \\
u(a)=u(b)=\vartheta_{\mathbb{R}^{n}}
\end{array}\right.
$$

has at least one generalized solution $u$ in $W^{2, s}\left([a, b], \mathbb{R}^{n}\right)$ such that $\left\|u^{\prime \prime}(t)\right\| \leq$ $m(t)$ a.e. in $[a, b]$.

Proof. Put $I:=f(Y), F(v):=f^{-1}(v)$ for every $v \in I$, and $G(t, x, z):=$ $\{g(t, x, z)\}$ for every $(t, x, z) \in[a, b] \times \mathbb{R}^{n} \times \mathbb{R}^{n}$. 
The multifunctions $F$ and $G$ satisfy all the assumptions of Theorem 3, in which $(\beta \beta)^{\prime}$ is used instead of $(\beta \beta)$.

This follows easily in the particular case when $f$ is constant.

If $f$ is not constant, then we can suppose that $\operatorname{int}(f(Y))$ is a non-empty open interval, and the only thing to prove is $(\alpha \alpha)$, which is equivalent to saying that, for every open set $\Omega \subset \mathbb{R}^{n}$, the set $f(\Omega \cap Y)$ has no isolated points. Suppose, on the contrary, that there exist $x_{0} \in \Omega \cap Y$ and $\varepsilon>0$ such that $] f\left(x_{0}\right)-\varepsilon, f\left(x_{0}\right)+\varepsilon\left[\cap f(\Omega \cap Y)=\left\{f\left(x_{0}\right)\right\}\right.$. Let $\Omega^{\prime}$ and $\Omega^{\prime \prime}$ be open subsets of $\mathbb{R}^{n}$ such that $x_{0} \in \Omega^{\prime} \subset \overline{\Omega^{\prime}} \subset \Omega^{\prime \prime} \subset \overline{\Omega^{\prime \prime}} \subset \Omega$. Taking into account $(\alpha \alpha)^{\prime}$, it is simple to verify that the sets $\left(\left(\Omega^{\prime} \cap Y\right) \times\right] f\left(x_{0}\right)-\varepsilon / 3, f\left(x_{0}\right)+$ $\varepsilon / 3[) \cap \operatorname{gr}(f)$ and $\left[(Y \times \mathbb{R}) \backslash\left(\left(\overline{\Omega^{\prime \prime}} \cap Y\right) \times\left[f\left(x_{0}\right)-\varepsilon / 2, f\left(x_{0}\right)+\varepsilon / 2\right]\right)\right] \cap \operatorname{gr}(f)$ are open in $\operatorname{gr}(f)$ and form a partition of $\operatorname{gr}(f)$, which is a contradiction.

Thus, the problem $\left(\mathrm{P}_{F \circ G}\right)$ has a generalized solution $u$ in $W^{2, s}\left([a, b], \mathbb{R}^{n}\right)$ such that $\left\|u^{\prime \prime}(t)\right\| \leq m(t)$ a.e. in $[a, b]$. The function $u$ is solution.

REMARK 7. We point out that, as the example on p. 227 of [18] shows, there are discontinuous functions $f$ satisfying the hypotheses $(\alpha),(\alpha \alpha)^{\prime}$ and $(\alpha \alpha \alpha)$ of Theorem 6 .

Remark 8. Theorem 6 improves Theorem 2.2 of [14], in which the continuity of $f$ and $g$ is required, $Y$ is a non-empty, compact, connected and locally connected subset of $\mathbb{R}^{n}$ and generalized solutions in $W^{2, \infty}\left([a, b], \mathbb{R}^{n}\right)$ can only be obtained.

Finally, we stress that Theorems 4 and 6 can give existence of solutions also for boundary value problems with no solutions in $W^{2, \infty}\left([a, b], \mathbb{R}^{n}\right)$ as the following example shows.

EXAmple 1. Consider the following boundary value problem:

$$
\left\{\begin{array}{l}
u^{\prime \prime}\left(2+\sin u^{\prime \prime}\right)=\frac{1}{4 \sqrt{t}}\left(|u|+\frac{3}{4}\right)\left(\frac{\left|u^{\prime}\right|+1}{2}\right), \\
u(0)=u(1)=\vartheta_{\mathbb{R}} .
\end{array}\right.
$$

Put $\|\cdot\|=\|\cdot\|_{1}=\|\cdot\|_{2}=|\cdot|$ and $c_{1}=c_{2}=1$. Theorem 4 or Theorem 6 can be used to prove existence of generalized solutions in $W^{2,1}([0,1], \mathbb{R})$.

In fact, put $Y:=\mathbb{R}, p:=s:=1$,

$$
m(t):= \begin{cases}1 /(2 \sqrt{t}) & \text { if } t \in] 0,1] \\ 0 & \text { if } t=0\end{cases}
$$

and $r:=\|m\|_{L^{1}([0,1], \mathbb{R})}$.

It is not difficult to verify that Theorem 4 can be used if we define, for every $(t, x, z, y) \in[0,1] \times \mathbb{R} \times \mathbb{R} \times Y$,

$$
f(t, x, z, y):= \begin{cases}y(2+\sin y)-\frac{1}{2 \sqrt{t}}\left(|u|+\frac{3}{4}\right)\left(\frac{\left|u^{\prime}\right|+1}{2}\right) & \text { if } t \in] 0,1], \\ y(2+\sin y) & \text { if } t=0 .\end{cases}
$$


In a similar way, Theorem 6 can be used if we define $f(y):=y(2+\sin y)$ for every $y \in Y$ and we put, for every $(t, x, z) \in[0,1] \times \mathbb{R}^{n} \times \mathbb{R}^{n}$,

$$
g(t, x, z):= \begin{cases}\frac{1}{2 \sqrt{t}}\left(|u|+\frac{3}{4}\right)\left(\frac{\left|u^{\prime}\right|+1}{2}\right) & \text { if } t \in] 0,1], \\ 0 & \text { if } t=0 .\end{cases}
$$

Nevertheless, $\lim _{t \rightarrow 0^{+}} u^{\prime \prime}(t)=\infty$ for every generalized solution of problem $(\mathrm{P})$, thus problem $(\mathrm{P})$ has no generalized solutions in $W^{2, \infty}([0,1], \mathbb{R})$.

\section{References}

[1] J. Appell, E. De Pascale, H. T. Nguyêñ and P. P. Zabreǔko, Multi-valued superpositions, Dissertationes Math. 345 (1995).

[2] Z. Artstein and K. Prikry, Carathéodory selections and the Scorza Dragoni property, J. Math. Anal. Appl. 127 (1987), 540-547.

[3] D. Averna, Lusin type theorems for multifunctions, Scorza Dragoni's property and Carathéodory selections, Boll. Un. Mat. Ital. (7) 8-A (1994), 193-202.

[4] G. Bonanno, Two theorems on the Scorza Dragoni property for multifunctions, Atti Accad. Naz. Lincei Rend. Cl. Sci. Fis. Mat. Natur. 83 (1989), 51-56.

[5] -, Differential inclusions with nonconvex right hand side and applications to implicit integral and differential equations, Rend. Accad. Naz. Sci. (detta dei XL) 20 (1996), 193-203.

[6] A. Bressan, Upper and lower semicontinuous differential inclusions: A unified approach, in: Controllability and Optimal Control, H. Sussmann (ed.), Dekker, New York, 1989, 21-31.

[7] C. Castaing, A propos de l'existence des sections séparément mesurables et séparément continues d'une multiapplication séparément mesurable et séparément semicontinue inférieurement, Sém. Analyse Convexe, Montpellier 1976, Exp. no. 6.

[8] F. S. De Blasi and G. Pianigiani, Solution sets of boundary value problems for nonconvex differential inclusions, Topol. Methods Nonlinear Anal. 1 (1993), 303-313.

[9] K. Deimling, Multivalued Differential Equations, de Gruyter Ser. Nonlinear Anal. Appl. 1, de Gruyter, Berlin, 1992.

[10] C. J. Himmelberg, Measurable relations, Fund. Math. 87 (1975), 53-72.

[11] J. B. Hiriart-Urruty, Images of connected sets by semicontinuous multifunctions, J. Math. Anal. Appl. 111 (1985), 407-422.

[12] A. Kucia, Scorza Dragoni type theorems, Fund. Math. 138 (1991), 197-203.

[13] S. A. Marano, Existence theorems for a multivalued boundary value problem, Bull. Austral. Math. Soc. 45 (1992), 249-260.

[14] -, On a boundary value problem for the differential equation $f\left(t, x, x^{\prime}, x^{\prime \prime}\right)=0$, J. Math. Anal. Appl. 182 (1994), 309-319.

[15] O. Naselli Ricceri and B. Ricceri, An existence theorem for inclusions of the type $\Psi(u)(t) \in F(t, \Phi(u)(t))$ and application to a multivalued boundary value problem, Appl. Anal. 38 (1990), 259-270.

[16] J. Oxtoby, Measure and Category, Springer, New York, 1971.

[17] B. Ricceri, Applications de théorèmes de semi-continuité inférieure, C. R. Acad. Sci. Paris Sér. I 295 (1982), 75-78.

[18] —, On multifunctions of one real variable, J. Math. Anal. Appl. 295 (1987), 225-236. 
[19] B. Ricceri and A. Villani, Openness properties of continuous real functions on connected spaces, Rend. Mat. 2 (1982), 679-687.

Dipartimento di Matematica ed Applicazioni Facoltà di Ingegneria

Università di Palermo

Viale delle Scienze

90128 Palermo, Italy

E-mail: averna@mbox.unipa.it
Dipartimento di Informatica, Matematica, Elettronica e Trasporti

Facoltà di Ingegneria

Università di Reggio Calabria

Via Graziella (Feo di Vito)

89100 Reggio Calabria, Italy

E-mail: bonanno@ing.unirc.it

Reçu par la Rédaction le 4.2.1998

Révisé le 8.6.1998 